\title{
Biventricular Thrombus Formation in Association with Acute Myocardial Infarction: Diagnosis by Two-Dimensional Echocardiography
}

\author{
Harold Z. Friedman, MD, and Andrew J. Buda, MD
}

Left ventricular thrombus formation is a well-described complication of acute myocardial infarction, and two-dimensional echocardiography is a reliable method for its detection. ${ }^{1.2}$ The echocardiographic detection of biventricular thrombi in this setting, however, has not previously been reported.

\section{CASE REPORT}

\section{Clinical Features}

A previously healthy 33-year-old man developed crushing substernal chest pain. An acute anterior myocardial infarction was diagnosed on the basis of clinical symptoms and pathologic $Q$ waves, ST-T wave changes, and a transient right bundle branch block on the electrocardiogram. Cardiac catheterization $5 \mathrm{~h}$ after the onset of pain revealed total proximal left anterior descending coronary artery occlusion and a $30 \%$ stenosis of the proximal circumflex artery. Left ventriculography showed globally depressed function, apical and septal dyskinesis, and severe anterolateral wall hypokinesis. Reperfusion of the occluded artery was achieved with balloon angioplasty, and a residual $80 \%$ stenosis and $18 \mathrm{~mm} \mathrm{Hg}$ translesional gradient remained. Intravenous nitroglycerin, lidocaine, and heparin, as well as oral dipyridamole $75 \mathrm{mg}$ TID; aspirin $324 \mathrm{mg}$ TID; and nifedipine $30 \mathrm{mg}$ TID were prescribed.

The early hospital course was complicated by hypotension necessitating the use of an intraaor-

From the Division of Cardiology, Department of Internal Medicine, University of Michigan Medical Center, Ann Arbor, Michigan. For reprints contact Andrew J. Buda, MD, Cardiology Division, University of Michigan Medical Center, W11607, Ann Arbor, MI 48109. tic balloon pump and temporary inotropic support. Repeat catheterization was performed on the third hospital day because of recurrent chest pain that was confirmed to be secondary to partial reocclusion of the left anterior descending artery. The stenosis was again reduced by angioplasty to $60 \%$, and the translesional gradient to less than $10 \mathrm{~mm}$ $\mathrm{Hg}$. There was no further chest pain. Hemodynamic recovery was complete, and intravenous heparin was stopped on the sixth hospital day. On the 10th day a radionuclide ventriculogram showed anterior, apical, and septal wall motion abnormalities and a left ventricular ejection fraction of $37 \%$.

\section{Echocardiography}

A two-dimensional echocardiogram was performed 18 days after infarction and demonstrated the previously noted wall motion defects as well as right and left ventricular masses. Both masses were highly echogenic, acoustically distinct from underlying myocardium, exhibited free intracavitary motion in systole and diastole, and were attached to the septum. Both of the masses were prominent in the parasternal long- and short-axis views (Fig. 1). Bilateral ventricular thrombi were diagnosed, and coumadin was prescribed. Eight weeks after infarction a repeat M-mode and twodimensional echocardiogram showed a thin akinetic septum and persistance of the biventricular thrombi despite therapeutic anticoagulation.

Catheterization at 2 months because of exertional chest pain revealed no change in the coronary anatomy. Moreover, a right atrial contrast injection with levophase ventriculography confirmed the presence of bilateral intraventricular thrombi. After 6 months, there was echocardiographic resolution of the left ventricular throm- 


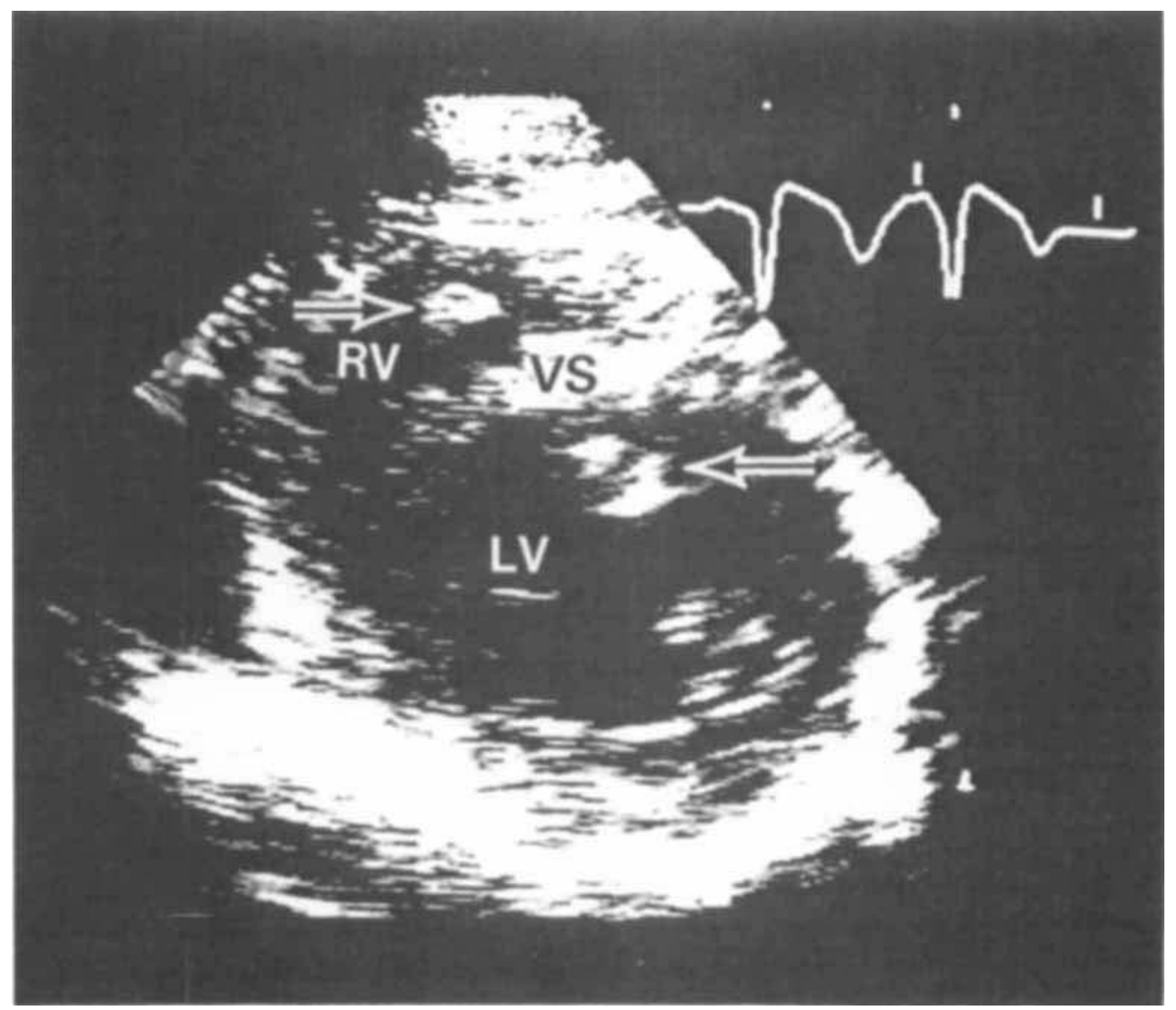

FIGURE 1. Short-axis stop of a two-dimensional echocardiographic parasternal short-axis projection at the papillary muscle level. Both left ventricular (arrow) and right ventricular (arrow) thrombi are visualized attached to the ventricular septum (VS). LV, left ventricular cavity; RV. right ventricular cavity.

bus but not of the right ventricular thrombus, and coumadin was continued. The patient remained well, and no embolic phenomena were noted during 1 year of follow-up.

\section{DISCUSSION}

\section{General Comments}

Left ventricular mural thrombi have been detected with two-dimensional echocardiography in $30 \%$ or more of patients with an acute transmural anterior myocardial infarction associated with an underlying apical wall motion abnormality: ${ }^{3-5}$ Large infarcts, as in this case, predispose to left ventricular thrombi, which can appear within days to months. ${ }^{3,4}$

Although the formation of right ventricular thrombus following acute myocardial infarction is known to occur, there have been relatively few published cases. Isner and Roberts reported a right ventricular thrombus on postmortem examination in $15(6 \%)$ of 237 patients with left ventricular wall infarction. ${ }^{6}$ Right ventricular thrombus occurred with a similar frequency in those with and those without an associated infarct of the right ventricular wall. Of their 97 patients with an anterior left ventricular infarct, a right ventricular thrombus that was not localized to free wall or septal surface occurred in $8 \%$. Two-dimensional echocardiographic demonstration of an infarct-related right ventricular thrombus, heralded by multiple pulmonary emboli, has been noted once.? Biventricular thrombi, confirmed at necropsy, were detected in one acute infarct patient with ${ }^{111}$ Inlabeled platelets. ${ }^{8}$ Biventricular thrombus formation in association with acute myocardial infarction has not previously been reported with echocardiography.

\section{Diagnosis}

The diagnosis of biventricular thrombi was based on a constellation of two-dimensional echocardiographic findings and supported by contrast cineangiography. Evaluation revealed a discrete mass of echoes in both ventricles that were distinct from the endocardium and moved freely in systole and 
diastole. ${ }^{1,9}$ In addition, an untoward clinical outcome for our patient was suggested by the findings of protruberence of thrombus into the left ventricular cavity and free mobility. These morphologic characteristics have been shown to identify those patients with the highest arterial embolic risk. $^{10,11}$

Right ventricular mural thrombi frequently develop after myocardial injury. Similar to left ventricular thrombi, right-sided thrombi appear following myocardial contusion and/or infarction and in all reported cases have been located in an area of asynergy. ${ }^{6,7,12,13}$ In our patient, septal dyskinesis, septal wall thinning, and right bundle branch block suggested that extensive septal damage accompanied the anterior-apical infarction. Thus, tissue necrosis through the interventricular septum and apex and the resulting asynergy may have promoted right ventricular thrombus formation.

The distinction of right ventricular thrombi from other intracavitary masses is possible with twodimensional echocardiography. Right atrial myxomas and thrombi may appear within the right ventricular cavity. ${ }^{14-16}$ Characteristically, these masses are seen intermittently when they prolapse across the tricuspid valve in diastole then suddenly disappear from the right ventricle in systole. Large tricuspid vegetations can appear in the ventricle during the cardiac cycle but should move with the leaflet to which they are attached..$^{16} \mathrm{Im}$ proper transducer angulation can distort normal echoes from the tricuspid annulus, chordae, papillary muscles, atrial septum, and anterior aortic root and create a mass-like effect within the right ventricle. Confusion of thrombi with objects other than tumors and vegetations such as hypertrophied muscle bands, pacing wires, thermodilution catheters, and prosthetic valves can best be avoided by familiarity with the characteristic location and appearance of these structures. ${ }^{17}$ Moreover, use of the clinical history and established criteria for the identification of left ventricular thrombi are essential for accurate diagnosis of right ventricular thrombi.

\section{Therapy}

The efficacy of immediate high-dose anticoagulation in preventing mural thrombus after acute anterior myocardial infarction has recently been noted. ${ }^{18}$ Early intervention with anticoagulants may reduce, but not necessarily eliminate, the development of mural clot. ${ }^{10.19,20}$ Chronic anticoagulation with coumadin, on the other hand, has been shown to reduce the incidence of embolic complications of infarct-related mural thrombi markedly. ${ }^{4.5}$ To date, however, no randomized, controlled study has defined the effects of anticoagulation on clot lysis or the value of treating asymptomatic thrombi that persist beyond several months. ${ }^{21}$

Current use of intravenous and intracoronary streptokinase therapy during acute infarction may be expected to reduce left ventricular thrombus formation by decreasing infarct size or by direct lysis of the fresh clot. The Western Washington intracoronary streptokinase trial, which randomized 83 patients to streptokinase or anticoagulation groups, showed a relatively low overall incidence of thrombus formation of $17 \%$ in anterior infarcts at 8 weeks. ${ }^{19}$ However, all thrombi were noted in the streptokinase-treated group, sug. gesting that acute thrombolysis may offer little additional benefit beyond that of anticoagulation. Thrombolytic therapy has also been used with ventricular thrombi that embolize despite therapeutic anticoagulation. Kremer et al was able to demonstrate echocardiographic dissolution or reduction in size of thrombi in seven of eight nonrandomized patients treated with urokinase. ${ }^{20}$ Thrombolysis in this setting does not appear to create additional embolic risk and offers an $r$ ternative for those patients who would otherwise be candidates for surgical thrombectomy.

It is of interest that the biventricular thrombi developed in this case despite the early and chronic administration of aspirin and dipyridamole. However, the antithrombotic effect of platelet antag. onists has been variable with chronic ventricular thrombi and has not been studied in the acute phases of infarction. ${ }^{22}$

\section{Implications}

We conclude that careful two-dimensional echocardiographic examination of the right ventricular chamber should be performed in patients suspected to have a thrombus complicating an acute myocardial infarction. Identification of ventricular thrombi using established criteria may allow prevention of serious pulmonary and systemic embolic complications with anticoagulant therapy.

\section{ACKNOWLEDGMENT}

We thank Sheree Wilson for her excellent secretarial assistance.

\section{REFERENCES}

1. Stratton JR, Lighty GW, Pearlman AJ, et al: Detection of left ventricular thrombus by two-dimen- 
sional echocardiography: Sensitivity, specificity and causes of uncertainty. Circulation 66:156, 1982.

2. Visser CA, Kan G, David GK, et al: Two dimensional echocardiography in the diagnosis of left ventricular thrombus. Chest 83:228, 1983.

3. Asinger RW, Mirell FL, Elsperger J, et al: Incidence of left ventricular thrombosis after acute transmural myocardial infarction. $N$ Engl $J$ Med 305:297, 1981.

4. Keating EC, Gross SA, Schlamowitz RA, et al: Mural thrombi in myocardial infarctions. Am $J$ Med 74:989, 1983.

5. Weinreich DJ, Burke JF, Pauletto FJ: Left ventricular thrombi complicating acute myocardial infarction. Ann Intern Med 100:789, 1984.

6. Isner JM, Roberts WS: Right ventricular infarction complicating left ventricular infarction secondary to coronary heart disease. Am J Cardiol 42:885, 1978.

7. Stowers SA, Leiboff RH, Wasserman AG, et al: Right ventricular thrombus formation in association with acute myocardial infarction: Diagnosis by 2-dimensional echocardiography. Am J Cardiol 52:912, 1983.

8. Ezekowitz MD, Kellerman DJ, Smith EO, et al: Detection of active left ventricular thrombosis during acute myocardial infarction using indium-III scintigraphy. Chest 86:35, 1984.

9. Asinger RW, Mikell FL, Sharma B, et al: Observations on detecting left ventricular thrombus with two dimensional echocardiography: Emphasis on avoidance of false positive diagnoses. Am J Cardiol 47:145, 1981.

10. Haugland JM, Asinger RW, Mikell FL, et al: Embolic potential of left ventricular thrombi detected by two-dimensional echocardiography. Circulation 70:588, 1984.

11. Visser CA, Kan G, Meltzer RS, et al: Embolic potential of left ventricular thrombus after myocardial infarction: A two-dimensional echocardiographic study of 119 patients. J Am Coll Cardiol 5:1276, 1985.
12. Kessler KM, Mallon SM, Bolooki H, et al: Pedunculated right ventricular thrombus due to repeated chest trauma. Am Heart J 102:1064, 1981.

13. Miller FA, Seward JB, Gersh BJ, et al: Two-dimensional echocardiographic findings in cardiac trauma. Am J Cardiol 50:1022, 1982.

14. Cameron J, Pohlner PG, Stafford EG, et al: Right heart thrombus: Recognition, diagnosis and management. J Am Coll Cardiol 5:1239, 1985.

15. Come PC: Transient right atrial thrombus during acute myocardial infarction: Diagnosis by echocardiography. Am J Cardiol 51:1228, 1983.

16. Come PC, Kurland AS, Vine HS: Two dimensional echocardiography in differentiating right atrial and tricuspid valve mass lesions. Am J Cardiol 44:1207, 1979.

17. Stern MJ, Cohen MJ, Fish B, et al: Clinical presentation and noninvasive diagnosis of right heart masses. Br Heart $J$ 46:552, 1981.

18. Nordrehaug JE, Johannessen KA, Von Der Lippe G: Usefulness of high-dose anticoagulants in preventing left ventricular thrombus in acute myocardial infarction. Am J Cardiol 55:1491, 1985.

19. Stratton JR, Speck SM, Caldwell JH: Late effects of intracoronary SK on regional wall motion, ventricular aneurysm and LV thrombus in MI: Results from the Western Washington randomized trial. $J$ Am Coll Cardiol 5:1023, 1985.

20. Kremer P, Fiebig R, Tilsner V, et al: Lysis of left ventricular thrombi with urokinase. Circulation 72:112, 1985.

21. Ezekowitz MD: Acute infarction, left ventricular thrombus and systemic embolization: An approach to management. J Am Coll Cardiol 5:281, 1985.

22. Stratton JR, Ritchie JL: The effects of antithrombotic drugs in patients with left ventricular thrombi: Assessment with indium-III platelet imaging and two dimensional echocardiography. Circulation 69: $561,1984$. 